\title{
PAULO FREIRE EN EL CHILE CONTEMPORÁNEO: ENFRENTANDO EL MIEDO A LA LIBERTAD
}

\author{
SANDra Araya-Crisóstomo ${ }^{1}$ \\ María Elena Infante Malachias ${ }^{2}$
}

\begin{abstract}
RESUMEN: En este ensayo, discutimos el impacto de la permanencia de Paulo Freire durante casi cuatro años y medio en Santiago de Chile. En ese tiempo, el educador se distanció de su experiencia brasileña y pudo reelaborar sus ideas pedagógicas al observar, vivenciar y cuestionar la situación social chilena en la década de 1960. En el año que se celebra el centenario de su nacimiento, traemos preguntas sobre el impacto del pensamiento de Freire para la educación en Chile y destacamos que movimientos populares, como la movilización de estudiantes de Enseñanza Media y el reciente "estallido social", son indicativos de que la obra de Freire es vigente, actual y necesaria para pensar, como sociedad chilena, los nuevos rumbos de la educación, ya que el momento actual es de enfrentar el miedo a la libertad.
\end{abstract}

Palabras-clave: Paulo Freire. Chile. Movimientos sociales. Educación. Libertad.

\section{PAULO FREIRE NO CHILE CONTEMPORÂNEO: ENFRENTANDO O MEDO À LIBERDADE}

RESUMO: Neste ensaio, discutimos o impacto da permanência de Paulo Freire por quase quatro anos e meio em Santiago do Chile. Naquela época, o educador se distanciou de sua experiência brasileira e pôde retrabalhar suas ideias pedagógicas observando, vivenciando e questionando a situação social chilena nos anos 1960. No ano em que se comemora seu centenário de nascimento, trazemos questionamentos sobre o impacto do pensamento de Freire para a educação no Chile e destacamos que movimentos populares, como a mobilização de estudantes do Ensino Médio e a recente "explosão social", são indicativos de que a obra de Freire é vigente, atual e necessária para pensar os novos rumos da educação, como sociedade chilena, já que o momento atual é de enfrentar o medo da liberdade.

Palavras-chave: Paulo Freire. Chile. Movimentos sociais. Educação. Liberdade.

\section{PAULO FREIRE IN CONTEMPORARY CHILE: FACING UP THE FEAR OF FREEDOM}

ABSTRACT: In this essay, we discuss the impact of Paulo Freire's stay for almost four and a half years in Santiago, Chile. At that time, the educator distanced himself

1.Universidad Católica del Maule - Facultad de Ciencias Básicas - Departamento de Biología y Química - Talca, Chile. E-mail: saraya@ucm.cl

2.Universidad de Sao Paulo - Escuela de Artes, Ciencias y Humanidades - Sao Paulo (SP), Brasil. E-mail: marilen@usp.br

Editora de Sección: Vicente Sisto 
from his Brazilian experience, and was able to rework his pedagogical ideas by observing, experiencing, and questioning the Chilean social situation in the 1960s. In the year that celebrates the centenary of his birth, we bring questions about the impact of the Freire's thought for education in Chile, as well as highlight that popular movements, such as the mobilization of High School students and the recent "social outbreak", are indicative that Freire's work is current and necessary to think, as a Chilean society, the new directions of education, since the current moment is to face the fear of freedom.

Keywords: Paulo Freire. Chile. Social movements. Education. Freedom.

\section{Introducción}

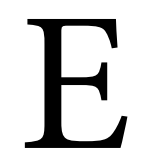

119 de septiembre de 2021 se celebran los 100 años del nacimiento de Paulo Freire, quien continúa siendo hasta el día de hoy, uno de los grandes referentes de la educación latinoamericana. Durante la década de 1970, período en el cual se acentuó la lucha por los derechos sociales en América Latina, sus ideas fueron recogidas de manera entusiasta por profesores y activistas sociales. Su obra Pedagogía del Oprimido, inserta en el movimiento de la pedagogía crítica o también llamada revolucionaria, fortaleció el desarrollo de movimientos sindicales y campesinos, así como también promovió el surgimiento de movimientos políticos de izquierda en diversos países del continente. Sus ideas influyeron en diversos procesos democráticos y en renovaciones pedagógicas en los sistemas educativos de países de América, Europa y África, y su principio del diálogo y pedagogía de la esperanza, entregaron una visión transformadora y progresista de la enseñanza, al promover una educación humanista destinada a fortalecer la formación de ciudadanos autónomos, insertos en su realidad social y con la voluntad de interferir y transformar la misma.

Su método de alfabetización campesina y sus ideas revolucionarias sobre la concientización y el proceso de liberación del hombre a través de la educación, lo llevaron a prisión tras el golpe militar en Brasil, siendo acusado de "subversivo". Tras su salida al exilio se establece inicialmente en Bolivia y posteriormente en Chile, donde sistematiza sus planteamientos en los libros Pedagogía del Oprimido y Educación para la Libertad, que dan cuenta de sus teorías sobre la opresión del sistema educativo y la dominación de la conciencia (RODRIGUEZ et al., 2007).

La obra de Freire permite múltiples reflexiones sobre conceptos como libertad, pedagogía crítica y diálogo, y requiere ser leído en el contexto de la "naturaleza profundamente radical de su teoría y de su práctica anticolonial” (GIROUX, 1993, p. 177). Para Freire, la educación supone que educador y educando comprendan de manera crítica su entorno, para luego reflexionar y dialogar, con el fin de actuar y transformar el mundo.

Sus aportes teóricos van más allá de su método de alfabetización y profundizan respecto al rol que la educación tiene en el ámbito político y social de los sujetos. Es por esta razón que antes de plantear la tesis de este ensayo, creemos que es de fundamental importancia revisar algunas de las ideas centrales del pensamiento de Paulo Freire y a la vez, destacar los principales hitos de su paso por Chile. Existe una vasta producción intelectual sobre la vida y obra de Freire en Brasil y alrededor de todo el mundo, sin embargo, a lo largo de este texto nos detendremos a reflexionar sobre el impacto del pensamiento freireano en la realidad contemporánea de Chile, principalmente después del llamado estallido social que se inició en octubre de 2019 y que tiene como resultado sin precedentes en la historia reciente del país, la convocatoria a una asamblea constituyente para elaborar una nueva carta magna de la nación, documento que contará con la representación de diversos grupos y sectores de la sociedad chilena que nunca antes habían sido contemplados ni escuchados. 
Dentro de los pilares teóricos de Freire que sustentan este ensayo, destacamos los conceptos de educación como un acto transformador, conciencia crítica como acción liberadora y la educación como acción dialógica. La concepción de la educación como acción transformadora, reconoce el acto educativo como una acción donde cambian juntos el sujeto y su mundo (PINTO, 2004). Para Freire toda práctica educativa es política, nunca es neutral, ya que esta acción, siempre favorece la dominación o la emancipación (FREIRE, 1997). En consecuencia, el docente debe adquirir un compromiso ético y político en el desarrollo de una sociedad más justa y no puede, por principios de su acción educadora, ser indiferente a la injusticia y la opresión. El fin de la educación es que los educandos sean capaces de transformarse a sí mismos y a su entorno para superar aquellos problemas socio-culturales, políticos y económicos que limitan y obstaculizan la realización plena del ser humano.

Respecto a la formación de una conciencia crítica en el hombre, esta se construye según Freire, al mismo tiempo en que este se forma como sujeto social. La construcción de la conciencia crítica consiste en descubrir la condición social e histórica de sí mismo y los condicionantes que lo oprimen, de modo que, para Freire, como seres humanos no somos en absoluto seres determinados, como el discurso conservador y de post verdades trata de defender, más bien somos seres condicionados (FREIRE, 1996). Cuando el ser humano se da cuenta de sus condicionamientos puede, a partir de ahí, desarrollar nuevos niveles de conciencia. En palabras de Freire "cuando la conciencia se torna crítica, el hombre es un ser integrado, situado y adaptado, y la historia y cultura cobran sentido. Cuando esto no ocurre sería apenas un ser acomodado o ajustado y la historia y cultura no tienen sentido" (FREIRE, 2013, p. 34).

En su primer libro escrito en Chile en 1965, La Educación como Práctica de la Libertad, Freire insiste en que no existe educación fuera de las sociedades humanas y, por lo tanto, no hay hombre en el vacío, el ser humano es un ser de relación (FREIRE, 2014), y es en esta relación que, el individuo puede trascender la intransitividad y asumir la transitividad crítica.

El concepto del estado de intransitividad del hombre defendido por Freire en su obra, indica que ese sujeto está más ocupado del ámbito biológico y, en consecuencia, le falta un sentido de vida en un plano histórico. Freire indica que, aunque utilice el concepto de intransitividad del hombre, este no significa que se encuentra encerrado en sí mismo. El hombre es un ser abierto, un ser de relación y lo que el autor pretende explicar con el término intransitivo es la limitación de la esfera de aprehensión de este sujeto humano. Es decir, su incapacidad de enfrentar desafíos situados fuera de su órbita vegetativa y es, en este sentido "que la intransitividad representa casi un descompromiso del hombre con su existencia" (FREIRE, 2014, p. 82). Cuando este hombre aumenta el poder de "dialogación" con su entorno y con otros hombres, comienza a transitivarse. Sus preocupaciones e intereses crecen a esferas más amplias y más históricas que sólo la esfera vital. En pleno siglo XXI podemos afirmar que la transitividad propuesta inicialmente por Freire puede extenderse a una toma de conciencia de que el hombre no es un simple consumidor como el capitalismo lo ha convencido en las últimas décadas. Esta conciencia transitiva inicialmente se presenta de forma ingenua, y se caracteriza por la simplicidad para evaluar los problemas, por el anacronismo de considerar todo tiempo pasado como mejor y por la subestimación del hombre común. A estos elementos, se le puede sumar la fragilidad de los argumentos, que en suma puede llevar a una conciencia fanatizada a la que el irracionalismo sectario nos conduce (FREIRE, 2014, p. 83). Es impresionante la actualidad y coherencia del pensamiento freireano que se muestra absolutamente vigente y necesario y, que se presenta en nuestro análisis paradojalmente, al mismo tiempo como denuncia y esperanza.

Finalmente, en este proceso de concientización, el ser humano puede llegar a la transitividad crítica mediado por una educación dialogal y activa centrada en la responsabilidad social y política y que, de acuerdo con Freire, se caracteriza por la profundidad en la interpretación de los problemas. En ese sentido, 
la responsabilidad es un dato existencial, y no puede ser incorporada a los sujetos sólo intelectualmente, sino también vivencialmente. De este modo y de acuerdo con Freire, esta realidad develada por la experiencia vivencial de los sujetos adquiere nuevas dimensiones de criticidad. Así, la concientización sólo puede llevarse a cabo por medio de una acción pedagógica que permita la humanización del hombre y lo ayude a reflexionar sobre sí mismo, sobre sus responsabilidades y su rol en la sociedad y la cultura.

Muchas de la críticas y acusaciones que Freire continúa recibiendo hasta el día de hoy se refieren a la visión de quienes confunden su pensamiento educacional con una concepción política partidaria determinada (WEFFORT, 2014), sin embargo, su campo de acción es la pedagogía y no la política, y en la transformación humana se generan cambios políticos. En cuanto a la educación como quehacer dialógico, este dice relación con la imposibilidad de concebir una acción humana sin la comunicación entre sujetos. Freire reconoce que el conocer y pensar surgen en relación con el otro, y que el conocimiento requiere ser expresado y comunicado (GADOTTI, 2006). El diálogo pedagógico es la posibilidad de situarse en el mundo colectivamente, de adquirir conocimientos que ayuden a transformar y humanizar al hombre. Esto implica, aprender a dialogar en la diversidad en busca del consenso, de saber negociar para llegar a acuerdos que permitan la convivencia en democracia.

El fortalecimiento y sistematización del pensamiento de Paulo Freire, se produjo en Chile durante la década de los 1960. La intensa acción política propia del período de Guerra Fría, consolidó su obra iniciada en la región nordeste de Brasil, donde en la época, de acuerdo con Weffort (2014) existían cerca de 15 millones de analfabetos para una población de 25 millones de habitantes. Su propuesta de alfabetización de adultos llevó a que la masa de votantes, por ejemplo, creciera de ochocientas mil a un millón trescientas mil personas en el estado de Pernambuco. Este proceso de alfabetización y concientización de los más excluidos fue violentamente interrumpido con el golpe militar de 1964 en Brasil. Luego de esto Freire viaja a la ciudad de La Paz en septiembre de 1964, pero un golpe militar en Bolivia lo llevó a radicarse en Santiago de Chile donde residió desde noviembre de 1964 hasta abril de 1969. La situación socio-política vivida en Chile en su estadía de cuatro años y medio, fue fundamental para estructurar su pensamiento político-pedagógico y le permitió reestudiar y sistematizar su método (ÁLVAREZ, 2011).

\section{Paulo Freire en Chile: Cuatro Años Productivos}

A su llegada a Chile en noviembre de 1964, Paulo Freire se incorpora a la propuesta gubernamental del entonces Presidente Eduardo Frei Montalva, "La Revolución en Libertad", que contaba con el apoyo ciudadano y de intelectuales progresistas de opción cristiana, bajo un clima de esperanza y cambio. Es así como, participa del INDAP (Instituto del Desarrollo Agropecuario), luego en el Programa Extraordinario de Educación de Adultos del Ministerio de Educación, con posterioridad en la Subsecretaría de Promoción Popular, para finalmente integrar el Instituto de Capacitación e Investigación en Reforma Agraria - ICIRA. Su impacto en el sistema educativo chileno, según Pinto (2004) se puede sintetizar en cuatro ámbitos:

1. Aporte en la alfabetización y educación en adultos: el Ministerio de Educación chileno creó en 1964 el Programa Nacional Extraordinario de Educación de Adultos, para el cual convoca como asesor especialista en alfabetización a Paulo Freire, con el objetivo de llevar a cabo la campaña de alfabetización de adultos en base a su método de la "palabra generadora" y que en Chile se denominó "Método Psicosocial de Alfabetización". Cabe señalar que la aplicación de su método en Chile, si bien se fundamentó en sus principios de alfabetización de adultos, 
adquirió características diferentes de acuerdo con el contexto chileno. Un ejemplo de ello, fue la investigación que Freire realizó para cambiar las "palabras generadoras" de acuerdo a la realidad y el lenguaje chileno.

2. Presencia teórica y experimental en el perfeccionamiento docente y la formación inicial de profesores: el discurso pedagógico humanista y el valor de la cultura como herramienta transformadora de Paulo Freire se estableció como bibliografía obligada en los procesos de actualización docente desarrollados a partir del año 1964 por el recién creado Centro de Perfeccionamiento, Experimentación e Investigación Pedagógica (CPEIP), con el fin de enfrentar la reforma educativa en preparación. De la misma forma, en los centros de educación superior y universidades chilenas donde se formaban futuros profesores, a partir de la reforma educativa de 1967, la lectura de Freire se tornó fundamental, a tal punto que años después, muchos de esos docentes formados al alero de la visión pedagógica freireana, formaron parte del movimiento de la Educación Popular.

3. Incorporación de la propuesta Freireana en establecimientos educativos de congregaciones religiosas: el perfil cristiano de Freire sumado al apoyo dado por la Iglesia Católica progresista, instaló como referente su pedagogía humanizadora, en órdenes religiosas como la Jesuita y de los Sagrados Corazones. Su visión sobre la pedagogía del oprimido se articuló con la doctrina de la escuela cristiana chilena y comprometió a muchos jóvenes en acciones educativas y de orden político, creando incluso nuevos referentes políticos de izquierda que contribuyeron a la base social y democrática presente en el gobierno de la Unidad Popular.

4. Desarrollo de la educación popular: se trató de la concientización de sectores populares para generar una sociedad más igualitaria, democrática y participativa. Su pensamiento pedagógico como proceso político liberador fue referente del movimiento de la educación popular en Chile y América Latina durante la década de 1970. Paulo Freire y su Pedagogía del Oprimido como proceso político humanizador, iluminaron el movimiento y permitieron generar resistencia a la imposición de la cultura del silencio a través de una práctica educativa concientizadora.

A nivel latinoamericano, su obra continúa siendo un gran referente en educación y ha sido inspiración de numerosas experiencias en países del Primer y Tercer mundo. No obstante, su legado e ideas parecen haber sido olvidadas por el sistema educativo chileno, tanto a nivel de políticas públicas, como de currículo educativo y por sobre todo en la práctica docente. Paulo Freire parece ser un personaje pasado de moda, solo útil para adornar con frases emblemáticas, discursos educativos de la autoridad de turno. A partir de los años 90 Freire fue completamente eliminado de las políticas públicas chilenas, aunque algunas experiencias de educación popular como las escuelas P-900 (CASTRO MONTECINO, 2003) permanecieron por algún tiempo como parte de los programas del Ministerio de Educación con el objetivo central de buscar la equidad y calidad de los aprendizajes cognitivos y socio afectivos en niños de escuelas básicas con bajo rendimiento escolar y en mayor riesgo social, a través del apoyo estatal en ejes claves como: el aprendizaje y satisfacción de estudiantes a través de monitores de la comunidad y el conocimiento mutuo y vínculo entre el grupo familiar y la escuela. Pese a estas iniciativas, el sistema educativo chileno fue adquiriendo un lenguaje propio del modelo neoliberal, con énfasis en la competitividad y en una visión de la educación como bien de consumo más que como un bien social y, se instaló en el sistema tanto la estandarización de resultados académicos en pruebas como el SIMCE (Sistema de Evaluación de la Calidad de la Educación) y la PTU (Prueba de transición Universitaria), como en la evaluación sistemática de los educadores y en la elaboración de un currículum nacional, anulando así toda libertad, diversidad y creatividad en la acción docente. 
Es indudable el legado de Freire en la educación chilena durante la década de 1960 y 1970, sobre todo en materia de alfabetización campesina y de educación popular, lo cual contribuyó a una mayor organización y participación de los sectores más postergados de la sociedad. Sin embargo, al momento de traer su pensamiento educacional al escenario educativo actual surgen las siguientes interrogantes: ¿Tendrán vigencia en el Chile contemporáneo las ideas y planteamientos de Paulo Freire?; ¿Habrá cabida para su pedagogía en un Chile dominado por el sistema neoliberal, el cual ha permeado todos los ámbitos de la vida humana, incluso el sistema educativo chileno?

La tesis del presente ensayo respecto a las interrogantes planteadas es que, a pesar de que su obra Pedagogía del Oprimido fue escrita hace ya más de 45 años atrás, los principios teóricos de Freire están hoy más vigentes que nunca en la sociedad chilena.

\section{La Actualidad de la Pedagogía Freireana en el Chile Contemporáneo}

El primer argumento que sustenta la tesis planteada en este ensayo son las movilizaciones estudiantiles que desde el año 2006, surgieron en Chile como una práctica liberadora que buscó romper con una herencia de la dictadura militar para así recuperar en parte la posibilidad de una educación equitativa y de calidad para todos sus ciudadanos. Lo que hay detrás de estas demandas estudiantiles es, principalmente, una crítica al modelo neoliberal, el cual enfatiza la eficiencia e ignora al ser humano. Freire fue un crítico del pensamiento neoliberal, y en ese sentido fue categórico en plantear que la educación no puede orientarse por el paradigma empresarial que busca controlar y naturalizar la desigualdad, sobre todo porque el acto pedagógico es por naturaleza democrático (GADOTTI, 2006). Un ejemplo de estos métodos de control, que no han generado los logros educativos esperados y que, por el contrario, han sido cuestionados por su desconexión con la realidad y por la falta de generación de una conciencia crítica en los estudiantes, es la prueba estandarizada SIMCE. Esta prueba que busca representar el aprendizaje a través de un puntaje, no considera la experiencia enriquecedora de un aprendizaje humanista y limita la acción transformadora del proceso educativo.

Una respuesta alternativa al modelo neoliberal y una posible solución a la demanda estudiantil respecto a un sistema educativo más igualitario, se puede encontrar en la teoría socio-político-educativa de Freire, quien plantea la construcción de conciencia histórica a través de una nueva modernidad, donde la educación vaya más allá de la instrucción e incorpore la dimensión cultural como parte de su proceso, con el fin de transformar vidas a través del desarrollo de una conciencia crítica sobre el mundo que los rodea (GADOTTI, 1997). En palabras de Freire:

La tarea de educar, sólo será auténticamente humanista en la medida en que procure la integración del individuo a su realidad nacional, en la medida en que le pierda miedo a la libertad, en la medida en que pueda crear en el educando un proceso de recreación, de búsqueda, de independencia y a la vez de solidaridad (FREIRE, 1968, p. 15).

Otro punto importante dentro de las demandas estudiantiles y del debate nacional en torno a la reforma educacional tiene relación con el concepto de calidad en la educación. La Ley General de Educación (LGE), en su artículo $2^{\circ}$ define educación de calidad como:

[...] un proceso de aprendizaje permanente que abarca las distintas etapas de la vida de las personas y que tiene por finalidad alcanzar su desarrollo espiritual, ético, moral, afectivo, 
artístico y físico, mediante la transmisión y el cultivo de valores, conocimientos y destrezas [...] capacitando a las personas para conducir su vida en forma plena, para convivir y participar en forma responsable, tolerante, solidaria, democrática y activa en la comunidad (EDUCACIÓN2020, 2015).

Si bien esta definición, se aleja del pensamiento neoliberal, donde calidad se confunde con competitividad, y se acerca más a la noción sostenida por Freire, deja en segundo plano algo que para el educador brasileño es clave, las relaciones sociales y humanas y, la necesidad de solidaridad, que nos harán competentes para enfrentar los problemas cotidianos junto con otros y no individualmente (GADOTTI, 1997). A esto se suma la falta de coherencia entre la definición de educación de calidad y sus parámetros a medir. Actualmente, los indicadores de calidad del sistema educacional chileno parecen más cercanos a una visión capitalista de la educación, ya que incluyen mediciones como: porcentaje de cobertura, logros educativos en pruebas estandarizadas como SIMCE o Programme for International Student Assessment (PISA) y nivel de inversión en la educación (MINEDUC, 2018). Sin embargo, una educación de calidad va más allá de la inversión económica y de la acumulación de conocimientos medidos en una prueba. Para Freire, conocer es establecer relaciones y no sólo memorizar mecánicamente contenidos escolares (GADOTTI, 2006). Los especialistas en educación coinciden con la mirada de Freire y reconocen que educar es crear vínculos, por ejemplo, el informe Jaques Delors de la UNESCO (1996) señaló cuales son los cuatro grandes pilares de la educación: aprender a aprender, aprender a hacer, aprender a vivir juntos y aprender a ser, sin embargo, estos no han sido incorporados de manera explícita por el sistema educacional chileno.

Un segundo argumento en favor de la tesis planteada, se vincula con la concepción del currículo. En la actualidad, las sociedades se ven enfrentadas a problemáticas de grandes dimensiones que exigen la participación de diferentes disciplinas para ser solucionadas (GARCÍA, 2013). Habitualmente se señala que la educación debe preparar al estudiante para la vida, lo que implica no abordar los problemas desde una perspectiva disciplinar, sino más bien, a través de un proceso educativo sistémico e interdisciplinar (ANDEREGG, 2003; STENTOFT, 2017).

Una contribución importante en este ámbito es la perspectiva curricular de Freire, para quien no es posible entender la pedagogía sin entender conceptos como transdisciplinariedad, transcurricularidad e interculturalidad, los cuales se constituyen en una exigencia de la propia naturaleza del acto pedagógico. Freire creía que la pedagogía era, en esencia, una ciencia transversal y, por tanto, insistía en la gestión colectiva del conocimiento social (GADOTTI, 2006). La educación que propone Freire es eminentemente transdisciplinar, ya que busca problematizar de manera crítica y colectiva, exigiendo una permanente postura reflexiva con el fin de buscar soluciones y transformar al ser humano y su entorno (FREIRE, 1968). En consecuencia, su visión del currículo interdisciplinar y su idea de escuela abierta al mundo, adquiere hoy más que nunca vigencia y relevancia. En este sentido, los círculos de cultura freireanos como dispositivos dialogales traen la perspectiva de un currículo etno constitutivo, donde de acuerdo con Macedo (2016) los diferentes actores son, teóricos legítimos de sus realidades porque llevan en sí capacidades como la posibilidad de describir, comprender, reflexionar, analizar y sistematizar sus situaciones y experiencias. Estos sujetos "producen puntos de vista, opiniones, definiciones de situaciones, sistematizaciones y legitimaciones simbólicas sobre la vida y el mundo, o sea, son creadores de etno métodos y, con ellos construyen realidades" (MACEDO, 2016, p. 33).

Si bien a nivel mundial, la perspectiva educativa interdisciplinar toma fuerza, con iniciativas como la del gobierno finlandés, de eliminar las asignaturas del currículo escolar y reemplazarlas por el enfoque educativo de proyectos, en Chile las autoridades educativas se han negado a tal innovación. La organización actual del currículo nacional en disciplinas ha distanciado a la escuela y al educando de la realidad social y 
cultural en la que está inserta. Incluso, la falta de integración y contextualización de los contenidos escolares ha influido de manera importante en el actual desinterés que presentan los estudiantes, en particular por áreas del conocimiento como las ciencias naturales (MEDINA-GALINDO, 2017). Un ejemplo claro de esta mirada conservadora fue la negativa que por dos años tuvo el Consejo Nacional de Educación (CNED), para aprobar el nuevo currículo de Ciencias Naturales en la formación general común, el cual buscaba abordar los contenidos desde una perspectiva interdisciplinaria y en base a la metodología de aprendizaje basado en proyectos $(\mathrm{ABP})$. Esta decisión refleja el paternalismo de un sistema educativo conservador y autoritario, que se niega a permitir que sus ciudadanos puedan salir de la transitividad ingenua hacia la conciencia crítica que les permita ejercer su autonomía y libertad, sin miedo. Privar a los estudiantes de una asignatura que relacione ciencia, con medioambiente y sociedad, les niega la posibilidad de comprender el mundo en que viven y les niega también la posibilidad de elaborar sus propios etno métodos.

Uno de los principales contraargumentos a la tesis planteada en este ensayo, y que refleja la perspectiva reproductiva presente en el modelo de escuela capitalista chileno, viene desde los planteamientos del sociólogo francés Pierre Bourdieu, quien señala que en la sociedad actual se impone una cultura dominante que se reproduce en la escuela de manera sistemática (BORDIEU; PASSERON, 1996). En primer término, Bourdieu concibe toda acción pedagógica como violencia simbólica, ya que impone de manera arbitraria la cultura de la clase dominante, aun cuando se presente a sí misma y a sus educadores como defensores de pedagogías libres, naturales o no represivas. En segundo término, Bourdieu plantea que la arbitrariedad en el currículo escolar y en la imposición de los profesores, nunca aparece de manera explícita, sino que resulta eficaz en la medida en que existe un desconocimiento de la verdad objetiva de la acción pedagógica (MOLINA, 2016). En palabras de Bourdieu y Passeron:

En la medida en que el éxito de toda acción pedagógica es función del grado en el que los receptores reconocen la autoridad pedagógica de la instancia pedagógica y del grado en que dominan el código cultural de la comunicación pedagógica, el éxito de una determinada acción pedagógica en una formación social determinada está en función del sistema de relaciones entre la arbitrariedad cultural que impone esta acción pedagógica, la arbitrariedad cultural dominante en la formación social considerada y la arbitrariedad cultural inculcada por la primera educación en los grupos o clases de dónde proceden los que sufren esta acción pedagógica (BORDIEU; PASSERON, 1996, p. 70).

Bajo esta premisa de la reproducción en el ámbito educativo, presentada por Bourdieu, no existiría en la actual sociedad de mercado, posibilidad alguna de dar cabida a una propuesta pedagógica como la de Paulo Freire, en donde la educación se concibe como un proceso de concientización, liberación y autonomía. Sin embargo, frente a las nuevas formas de exclusión, discriminación y opresión que operan en el siglo XXI, se han levantado las más diversas expresiones de protesta. Movimientos como el estudiantil, el feminista universitario o el indígena, sumado a las movilizaciones de la clase media contra el sistema financiero y de pensiones, son señales claras y esperanzadoras de un pueblo que se levanta en torno a una acción colectiva. Estos nuevos actores sociales requieren de un nuevo paradigma socio-educativo que forme a sus dirigentes y bases sociales en una relación dialógica que, dé voz a todas las personas, a mujeres, indígenas, jóvenes e inmigrantes, por medio de una educación freireana basada en la acción reflexiva, la participación y el pensamiento crítico.

En su libro Pedagogía de la Esperanza, Freire realiza una relectura de su manuscrito, elaborado en Chile y publicado, en 1970, en Inglés: “ [...] viviendo la intensidad de la experiencia de la sociedad chilena, de mi experiencia dentro de aquella experiencia, que me hacía re-pensar siempre la experiencia brasileña, 
cuya memoria viva llevé conmigo para el exilio, fue que escribí Pedagogía del Oprimido entre 1967 y 1968” (FREIRE, 1992, p. 53). Así como en la época en que Freire estuvo en Chile, se necesita construir un proyecto de sociedad, donde el ser humano se desarrolle en plenitud tanto individual como colectivamente, priorizando lo social sobre lo económico (GADOTTI, 2006). En el momento actual, donde el modelo neoliberal con su concepción bancaria, se impone en lo político y económico, impulsando una visión de sociedad donde no tienen cabida las utopías y las luchas sociales parecen infructuosas, Freire nos da esperanza y nos devuelve la capacidad de soñar con una sociedad más humana y más justa, de la mano de una educación crítica, democrática, comprometida y liberadora, que hace pensar "otro mundo posible".

En su Pedagogía del Oprimido Freire afirma que los oprimidos tienen miedo a la libertad, miedo a no saber qué hacer con ella una vez que fueron sistemáticamente mandados a hacer las cosas de manera heterónoma, sin derecho a pensar, a crear lo nuevo, a equivocarse. (FREIRE, 1968). En este sentido vemos con profundo respeto y alegría que el llamado "estallido social" que comenzó en Chile en octubre de 2019, es la respuesta esperanzadora de un pueblo que después de muchos años de injusticia y opresión está perdiendo el miedo a la libertad. El trecho que destacamos a continuación fue escrito por Paulo Freire en Santiago de Chile, en la primavera de 1965:

Se encontraba entonces el pueblo, en la fase anterior de cierre de nuestra sociedad, inmerso en el proceso. Con el quiebre y la entrada de la sociedad en la época del tránsito, emerge. Si en la inmersión era apenas espectador del proceso, en la emersión descruza los brazos, renuncia a la expectación y exige la injerencia. Ya no se satisface en asistir. Quiere participar. Su participación que implica una toma de consciencia apenas y no una concientización - desarrollo de la tomada de consciencia - amenaza a las élites detentoras de privilegios. Se agrupan estas entonces para defenderlos. En un primer momento reaccionan espontáneamente. En una segunda fase, se dan cuenta claramente de la amenaza contenida en la toma de conciencia de parte del pueblo. Se reúnen. Atraen para sí mismos los "teóricos" de "crisis", como, de manera general, llaman el nuevo clima cultural. Crean instituciones asistenciales, que se transforman en asistencialistas. Y, en nombre de la libertad "amenazada", repelen la participación del pueblo, defienden una democracia sui generis en la que el pueblo es un enfermo, a quien se le aplican remedios. Y su enfermedad está precisamente en tener voz y participación [...] la salud para esta extraña democracia, está en el silencio del pueblo, en su quietud (FREIRE, 2014, p. 75-76).

La primera dama del actual gobierno de Chile, Cecilia Morel, expresó por mensaje de audio en octubre de 2019 y que fue divulgado por la prensa nacional e internacional que "Vamos a tener que disminuir nuestros privilegios" y calificó las multitudinarias manifestaciones como una "invasión extranjera, alienígena". Estas afirmaciones no nos sorprenden y nos llevan a darnos cuenta que el legado del profesor Paulo Freire no podría ser más relevante y actual. Sus proféticas palabras nos sitúan como protagonistas del caminar humano y nos hacen oír nuevamente su voz serena que, dando coraje a los oprimidos del mundo, insiste en decirnos que la historia es un tiempo de posibilidades porque como seres humanos somos seres inacabados.

Al terminar de escribir este texto somos sorprendidas por un hecho histórico que abre un nuevo rumbo en la historia de Chile, el día 4 de julio de 2021 una mujer mapuche fue elegida como presidente de la Convención Constitucional que tendrá la tarea de reelaborar la constitución. Elisa Loncon Antileo, mapuche, chilena doctora y docente universitaria con sus palabras -Mañum pu lamngen, marichiweu! (¡Gracias hermanos y hermanas, venceremos!) nos muestra que las palabras de Freire eran ciertas y que con un pueblo que asume críticamente su propia historia y sus dolores podemos decir que, como chilenas y chilenos, comenzamos a desafiar y enfrentar con coraje y esperanza el miedo que le teníamos a la libertad. 


\section{Referencias}

ANDER-EGG, E. Interdisciplinariedad en educación. Buenos Aires: Editorial Magisterio, 2003.

ÁlVAREZ, A. Influencia del pensamiento de Paulo Freire en Chile: ideas para un debate. Revista Perspectivas: Notas sobre Intervención y Acción Social, Santiago de Chile, n. 22, p. 215-237, 2011. https:// doi.org/10.29344/07171714.22.458

BOURDIEU, P.; PASSERON, J.-C. La reproducción. Ciudad de México: Editorial Laia S.A, 1996. 288 p.

EDUCACIÓN2020. Qué es educación de calidad. Educación2020.cl, 2 mar. 2015. Disponible en: http:// educacion2020.cl/noticias/que-es-educacion-de-calidad/. Acceso en: 13 mayo 2021.

CASTRO MONTECINO, L. I. El Programa de las 900 Escuelas y el impacto real de las escuelas. 2003. Dissertação (Mágister en Politica y Gestión Internacional) - Instituto de Investigación y Desarrollo Educacional, Universidad de Talca, Talca, 2003. Disponible en: http://dspace.utalca.cl/bitstream/1950/5852/1/ castro_montecino.pdf. Acceso en: 22 sep. 2021.

FREIRE, P. Pedagogia do oprimido. Rio de Janeiro: Paz e Terra, 1968. 246 p.

FREIRE, P. Pedagogia da esperança: um reencontro com a pedagogia do oprimido. São Paulo: Paz e Terra, 1992. $127 \mathrm{p}$.

FREIRE, P. Pedagogia da autonomia: saberes necessários à prática educativa. São Paulo: Paz e Terra, 1996. $148 \mathrm{p}$.

FREIRE, P. La educación en la ciudad. Buenos Aires: Editorial Siglo XXI, 1997. 167 p.

FREIRE, P. La educación como práctica de la libertad. Buenos Aires: Editorial Siglo XXI, 2013. 150 p.

FREIRE, P. Educação como prática da liberdade. São Paulo: Paz e Terra, 2014. 189 p.

GADOTTI, M. Lições de Freire. Revista da Faculdade de Educação, São Paulo, v. 23, n. 1-2, 1997. https:// doi.org/10.1590/S0102-25551997000100002

GADOTTI, M. La pedagogía de Paulo Freire y el proceso de democratización en el Brasil: algunos aspectos de su teoría, de su método y de su praxis. In: AYUSTE, A. (org.). Educación, ciudadanía y democracia. Barcelona: Octaedro Editorial, 2006.

GARCÍA, R. Investigación interdisciplinaria de sistemas complejos: lecciones del cambio climático. Interdisciplina, Ciudad de México, v. 1, n. 1. 2013. https://doi.org/10.22201/ceiich.24485705e.2013.1.46545

GIROUX, H. La escuela y la lucha por la ciudadanía. México: Ediciones Siglo XXI, 1993.

MACEDO, R. A teoria etnoconstitutiva do currículo: teoria-ação e sistema curricular formacional. Curitiba: Editora CRV, 2016. 162 p.

MEDINA-GALINDO, E. Conceptos y fenómenos: propuesta de reforma del plan de estudios en el bachillerato. Revista Científica, Bogotá, p. 13-22, 2017. https://doi.org/10.14483/udistrital.jour.RC.2017.27.a2

MINEDUC [MINISTERIO DE EDUCACIÓN]. Indicadores de la educación en Chile, 2010-2016. Centro de Estudios MINEDUC, 2018. 228 p. 
MOLINA, M. La sociología del sistema de enseñanza de Bourdieu: reflexiones desde América Latina. Cadernos de Pesquisa, São Paulo, v. 46, n. 162, p. 942-964, 2016. https://doi.org/10.1590/198053143615

PINTO, R. Paulo Freire: un educador humanista cristiano en Chile. Pensamiento Educativo, Santiago de Chile, v. 34, p. 234-258, 2004. https://doi.org/10.7764/PEL.34.1.2004

RODRIGUEZ, L. et al. Paulo Freire. Una pedagogía desde América Latina. Ciencia, Docencia y Tecnología, Entre Ríos, v. 28, n.34, p. 129-171. 2007.

STENTOFT, D. From saying to doing interdisciplinary, learning: is problem-based learning the answer? Active learning in Higher Education, Thousand Oaks, v. 18, n. 1, p. 51-61, 2017. https://doi. org/10.1177/1469787417693510

UNESCO [UNITED NATIONS EDUCATIONAL, SCIENTIFIC AND CULTURAL ORGANIZATION]. La educación encierra un tesoro. Informe a la UNESCO de la Comisión Internacional sobre la Educación para el siglo XXI presidida por Jaques Delors. Madrid: Ediciones Santillana, 1996.

WEFFORT, F. C. Educação e política: reflexões sociológicas sobre uma pedagogia da liberdade. In: FREIRE, P. Educação como prática da liberdade. São Paulo: Paz e Terra, 2014.

\section{Contribución de las Autoras}

Problematización y Conceptuación; Araya-Crisóstomo S y Infante-Malachias ME; Metodología; Araya-Crisóstomo S y Infante-Malachias ME; Análisis; Araya-Crisóstomo S y Infante-Malachias ME; Redacción; Araya-Crisóstomo S y Infante-Malachias ME.

\section{Sobre las Autoras}

Sandra Araya Crisóstomo es Profesora de Biología y Magíster en Didáctica de las Ciencias Experimentales por la Universidad Católica del Maule (UCM, Chile). Actualmente, es Doctoranda por la Universidad de Concepción (UDEC, Chile). Es académica de la Facultad de Ciencias Básicas de la UCM. Su área de investigación es la Didáctica y Enseñanza de las Ciencias.

María Elena Infante-Malachias es Doctora y Magíster en Ciencias Biológicas por la Universidade Estadual de Campinas (Unicamp, Brasil). Realizó su Postdoctorado en Educación por la Universidade de São Paulo (USP, Brasil). Es docente de la Escola de Artes, Ciências e Humanidades (EACH-USP, Brasil) y directora del Grupo de Pesquisa em Ensino de Ciências e Biologia do Conhecimento de la misma institución. Su área de investigación es la Enseñanza de las Ciencias y la Formación de Profesores en las perspectivas de Paulo Freire y de Humberto Maturana. 